\title{
Creating Ultrafast Electron Pulses Using a Microfabricated Laser-Triggered Beam Blanker
}

\author{
I.G.C. (Gerward) Weppelman ${ }^{1}$, C.T.H. Heerkens ${ }^{1}$, R.J. Moerland ${ }^{1}$, J.P. Hoogenboom ${ }^{1}$, P. Kruit ${ }^{1}$ and \\ R.F.C. van $\mathrm{Tol}^{2}$ \\ 1. TU Delft, Imaging Physics, Delft, The Netherlands. \\ 2. TU Delft, DEMO, Delft, The Netherlands.
}

Ultrafast electron pulses enable imaging of transient dynamics with nanometer and femtosecond resolution. Such pulses are typically created by illuminating a flat photocathode or a cold-field emitter with femtosecond laser pulses. [1,2] Using a cold field emitter, an ultrafast Scanning Electron Microscope (USEM) has been realized enabling to image charge carrier diffusion in time and spatial domain. [3] Another approach to achieve ultrafast electron pulses is to use beam blankers using $\mathrm{GHz}$ magnetic or electric fields with resonant cavities, hence such blankers are relatively large. [4,5] Recently, we presented the concept of a laser-triggered beam blanker for use in regular EMs that allows easy and quick switching between continuous-beam and ultrafast modes of operation.[6] In this concept we propose to use a micro fabricated beam blanker controlled by a photoconductive switch, which is illuminated with femtosecond laser pulses, shown in Figure 1. Using COMSOL simulations we show that our design can in principle deliver sub $100 \mathrm{fs}$ electron pulses by limiting the dimensions of the device to micrometer scales. We will also discuss the performance enhancement of the Auston switch using plasmonic contact electrodes. [6] We fabricated and integrated the beam blanker and a LT-GaAs photoconductive switch in one micrometer scale device. The ultrafast blanker is built on top of a LTGaAs chip. Also a stick is designed containing the blanker into the beam line of a commercial Quanta FEG SEM. Finally we will present our progress towards experimental realization and characterization of an ultrafast beam blanker, showing laser-triggered electron beam deflection. With a single photon detector we show that electron pulses triggered with a femtosecond laser pulse are created, as indicated in Figure 2.

\section{References:}

[1] A.H. Zewail, Science 328 (2010), p. 187.

[2] P. Hommelhof et. al, Phys. Rev. Lett. 96 (2006), 077401.

[3] D. Yang et al, PNAS 107 (2010), p. 14993.

[4] K. Ura et al, J. Electron Microsc. 27 (1978), p. 247.

[5] A. Lassise et al, Rev. Sci. Instrum. 83 (2012), 043705.

[6] I.G.C. Weppelman et al, Ultramicroscopy. 184 (2018).

[7] C.W. Berry et al, Nat. Communications 4 (2013), p. 1622. 


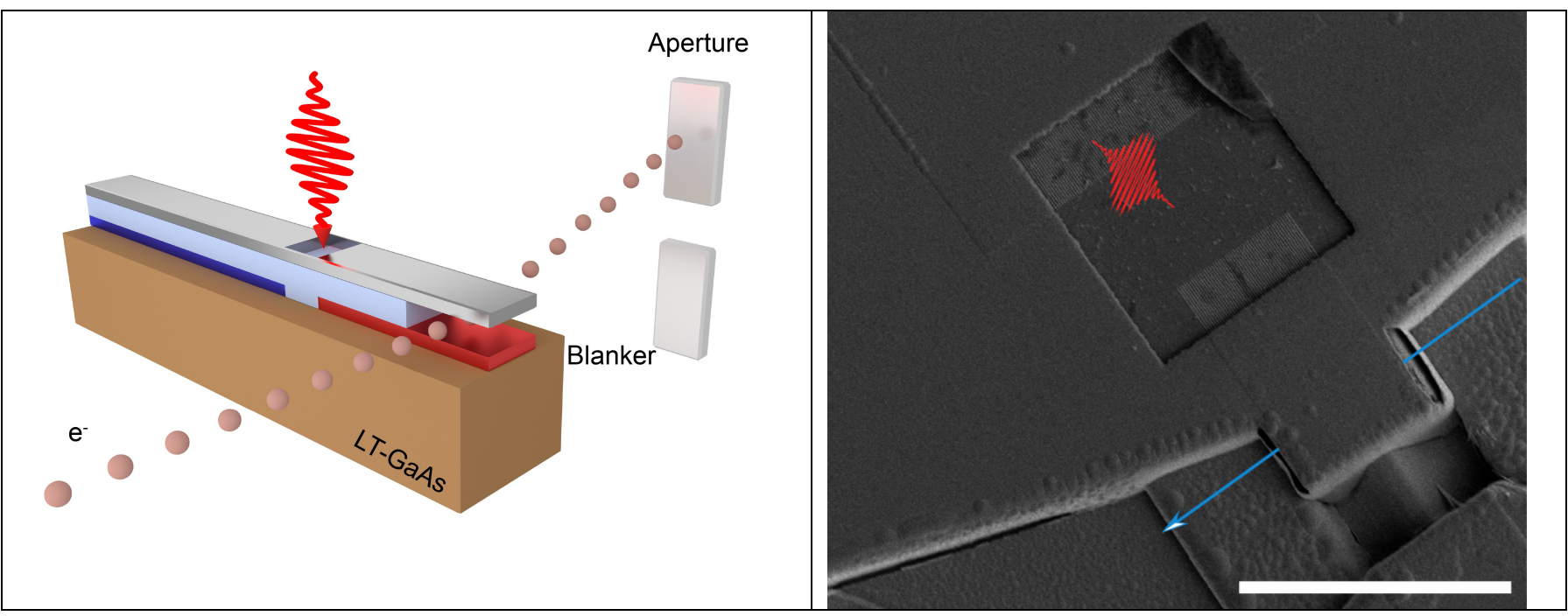

Figure 1. (left) Schematic indication of our UFB concept: a photoconductive switch is connected to an electrostatic beam blanker, the electron beam is deflected and intercepted by an aperture, the colors of the electrodes indicate $+10 \mathrm{~V}$ (blue), negative $-10 \mathrm{~V}$ (red) and ground (grey). SEM image of the MEMS UFB. The photoconductive switch is indicated with a red laser pulse, the electrodes consist of a grating to improve the photoconductivity. The blue arrow indicates the trajectory of the electron beam, the region where the line is intercepted is the region where it travels through the deflector unit. The scale bar is $20 \mu \mathrm{m}$.

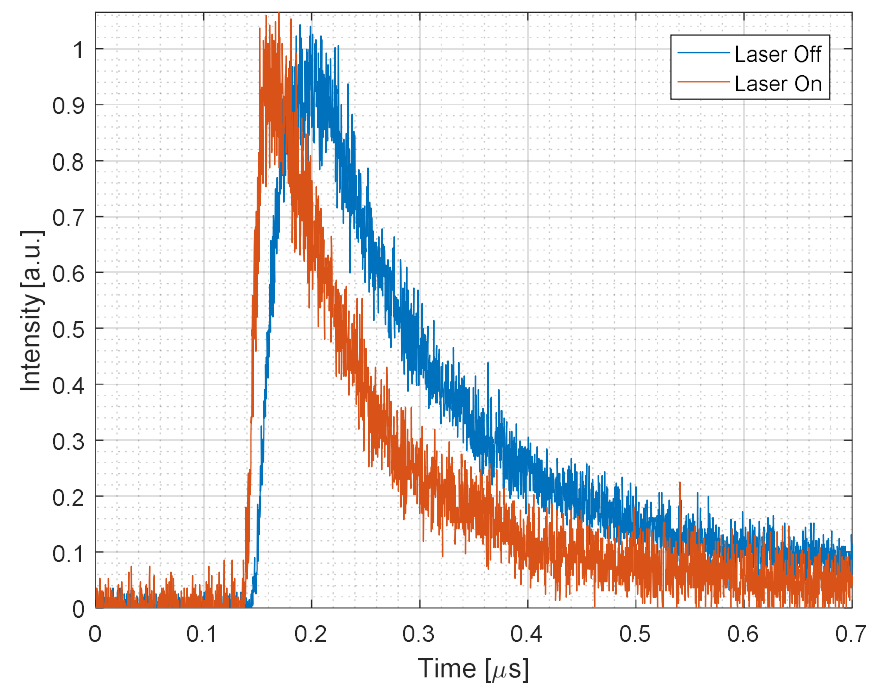

Figure 2. Normalized count rate as function of time in case with the laser off (blue line) and with a pulsed10 $\mathrm{mW}$ laser beam illuming the photoconductive switch (red line). 